\title{
Research on Consumption Rules of Air Material in Typical Equipment
}

\author{
Xiaopei Shi ${ }^{1}$, Shenyang Liu ${ }^{2}$, Qian Zhu ${ }^{2}$, Guangxu Luo ${ }^{2}$ \\ ${ }^{1}$ Department of Air Material Management, Air Force Logistics College, Xuzhou 221000, China \\ ${ }^{2}$ Department of Aviation Four Stations, Air Force Logistics College, Xuzhou 221000, China
}

Keywords: typical equipment; normal distribution; air material in typical equipment consumption

\begin{abstract}
Nearly all the segments of air material in typical equipment include acquisition, storage, supplying and management, which have close connections with the air material in typical equipment consumption information. The air material in typical equipment consumption rule has a great significance on all the segments about air material in typical equipment include acquisition, storage, supplying and management and improving the scientificity of air material in typical equipment support. Through an analysis of the maintenance method of air material in typical equipment whose life submits to normal distribution in some typical equipment, this paper has established consumption models of air material in typical equipment whose life submits to normal distribution according to stochastic processes and probability theory and mathematical statistics. Applicability of the models are given by way of a numerical example. The models provide a theoretical basis for calculating reserves of air material in typical equipment scientifically and have a vital important guiding significance.
\end{abstract}

\section{INTRODUCTION}

With the application of high-tech and information technology, different kinds of equipment have become more and more complex in recent years. At the same time, different maintenance strategies also tend to be applied for different components of equipment, making it difficult to grasp the law of equipment air material in typical equipment consumption and leading to heavier workload of air material in typical equipment consumption forecasting. To meet the needs of equipment maintenance, an organization need to store a certain variety and quantity of air material in typical equipment in advance. To ensure that air material in typical equipment stored in the organization is of reasonable quantity and good quality and can timely and reliably guarantee the equipment maintenance needs, a scientific and valid method of air material in typical equipment consumption forecasting must be given. If the storage capacity of air material in typical equipment is too small, the equipment's successful completion of the training mission can not be guaranteed; if the storage capacity of air material in typical equipment is too much, it will cause overstock which affects economic benefit of the components.

Many scholars have conducted in-depth studies of methods of air material in typical equipment consumption forecasting (LIU Xiao-qun, 2005). They also have done scientific researches on spare parts consumption forecasting (LI Yu-feng, 2014). Through the analysis of the previous literature, it can be found there are few undertaken research works of the methods of air material in typical equipment forecasting based on a variety of maintenance strategies (WANG Liang, 2005).

A certain type of equipment is maintained with a combination of inspection and regular maintenance (WANG Tie-ning, 2009). Within one year, in the normal training phase of the equipment, the equipment can be inspected and after the end of the training, regular maintenance of the equipment can be carried out (LI Yufeng, 2014). There may exist two maintenance strategies called "non-replacement" and "condition based replacement" in the inspection of the air material in typical equipment; there may exist three maintenance strategies called "non-replacement", "certain replacement" and "condition based replacement" at the regular maintenance of equipment. "Non-replacement" after inspection refers to the strategy not to replace air material in typical equipment after failure or problem is found in inspection when the replacement condition is not available; "condition based replacement" after inspection refers to the strategy to replace air material in typical equipment after failure or problem is found in inspection when the replacement condition is available; "non-replacement" after regular maintenance refers to the strategy not to replace air material in typical equipment after failure or problem is found in regular maintenance when the replacement condition is not available; “certain replacement" after regular maintenance refers to the strategy to replace air material in typical equipment no matter the component is damaged nor not during regular maintenance when the replacement condition is available; "condition based replacement" after regular maintenance refers to the strategy to replace air material in typical equipment if the service time of the air material in typical equipment exceeds a predetermined value during the regular maintenance when the replacement condition is available (LI Yu-feng, 2014).

The determination of the storage capacity of air material in typical equipment should be based on the consumption law of the air material in typical equipment. How to scientifically predict the air material in typical equipment consumption of the equipment under the circumstance of various maintenance is a key issue in this text. 


\section{INFLUENCE FACTORS OF AIR MATERIAL CONSUMPTION RULE}

The maintenance strategy of equipment components determines how the component will be replaced in the next year, therefore it is closely associated with the air material in typical equipment consumption generated by the equipment in the next year. In addition, the four main factors including components lives, remaining lives of components, the age of the equipment and next year's operation time have a direct impact on the air material in typical equipment consumption generated by equipment in the next year [6].

(1) Next year's operation time. Under the condition that the other factors that influence air material in typical equipment consumption are unchanged, the length of equipment operation time determines the quantity and amount of air material in typical equipment consumption. The longer the next year's operation time is, the larger the quantity and amount of air material in typical equipment consumption is; the shorter the next year's operation time is, the smaller the quantity and amount of air material in typical equipment consumption is.

(2) The age of the equipment. The length of the age of the equipment decides the equipment maintenance level in the next year. When different levels of maintenance are undertaken for the equipment, the maintenance strategy adopted by the same component may not be the same. Therefore, determination of the air material in typical equipment maintenance strategy for next year should be based on the age of the equipment.

(3) Remaining lives of air material in typical equipment. Remaining lives of air material in typical equipment refer to the interval time from the counting date after the air material in typical equipment has been working for some time to occurrence of malfunction. Remaining lives of air material in typical equipment are also random variables. If the air material in typical equipment is replaced at the previous year's regular maintenance, then when the equipment is trained in the next year, the air material in typical equipment can be seen as a new product whose remaining life is equal to its life; if the air material in typical equipment is replaced at the previous year's regular maintenance, then in determination of the remaining lives of air material in typical equipment, the period that the air material in typical equipment has been used should be fully considered.

(4) Air material in typical equipment lives. Air material in typical equipment lives refer to the interval time from usage of air material in typical equipment as new products ( 0 time) to occurrence of malfunction. Air material in typical equipment lives are random variables, and hence the time when the air material in typical equipment has malfunction is uncertain. However, under normal circumstances, the longer the average air material in typical equipment lives are, the smaller the air material in typical equipment consumption quantity and amount generated by the equipment in the next year is.

\section{MATERIAL CONSUMPTION MODELS}

Air material in typical equipment consumption affected by many outside factors is stochastic and has the complicated nonlinear character, but in air material in typical equipment consumption predicting practice, the small sample data of air material in typical equipment consumption or the reliability of the units is the only available information that could be used to forecast air material in typical equipment consumption by variety of reasons. If we apply the traditional statistical forecasting models in the project practice, the forecasting results of the traditional statistical forecasting models and statistical values are discrepan because the theoretical defects exists in the models and the data amount of air material in typical equipment consumption is small. The predictive values calculated by the air material in typical equipment consumption forecasting models that are proposed in this paper based on probability theory and mathematical statistics and the stochastic process theory are close to the statistical values [8].

No matter the working lives of identical units in a series system fit exponential distribution, normal distribution or gamma distribution, the working lives of the identical units are independent [9]. So first of all, we should do research on the air material in typical equipment consumption law of one unit, then we can master the air material in typical equipment consumption law based on the air material in typical equipment consumption models of the series system.

Assuming the units in the series system are both new products at the very start, we can gain the general formulas of air material in typical equipment consumption of the series system based on renewal theory.

The probability of the air material in typical equipment consumption number $y_{1} \geq n$ of one unit during the period from 0 to $t$ is

$$
P\left(y_{1} \geq n\right)=P\left(\sum_{i=1}^{n} T_{i}<t\right)
$$

The probability of the air material in typical equipment consumption number $y_{1}=n$ of one unit during the period from 0 to $t$ is

$$
\begin{aligned}
P\left(y_{1}=n\right)=P\left(y_{1}\right. & \geq n)-P\left(y_{1} \geq n+1\right) \\
& =P\left(\sum_{i=1}^{n} T_{i}<t\right)-P\left(\sum_{i=1}^{n+1} T_{i}<t\right)
\end{aligned}
$$

\section{APPLICATIONS}

Through the investigation of systems in the typical equipment and the analysis of statistical failure data, we could find that the working life of the air material in typical equipment fits normal distribution, the mathematical expectation is $u=2 \times 10^{3} \mathrm{~h}$ and the variance is $\sigma^{2}=300^{2} \mathrm{~h}^{2}$. If any the air material in typical equipment in the series systems is broken-down, 
the accendants will replace the air material in typical equipment immediately. Analyze the air material in typical equipment consumption laws and forecast the average number of the air material in typical equipment $\mathrm{s}$ consumption of the series systems in 20,000 hours.

The probability of the spare parts consumption number $y=0$ in the series system during the period from 0 to $t$ is

$$
\begin{array}{r}
P(y=0)=1-\Phi\left(\frac{t-u}{\sigma}\right)=1-\Phi\left(\frac{20000-2000}{300}\right) \\
=1-\Phi(60)
\end{array}
$$

The probability of the spare parts consumption number $y=n(n \geq 1)$ in the series system during the period from 0 to $t$ is

$$
\begin{array}{r}
P(y=n)=\Phi\left(\frac{t-n u}{\sqrt{n} \sigma}\right)-\Phi\left(\frac{t-(n+1) u}{\sqrt{n+1} \sigma}\right) \\
=\Phi\left(\frac{20000-2000 n}{300 \sqrt{n}}\right)-\Phi\left(\frac{20000-2000(n+1)}{300 \sqrt{n+1}}\right)
\end{array}
$$

The average number of the spare parts consumption of the series system in 20,000 hours is

$$
\bar{y}=\sum_{k=1}^{+\infty} \Phi\left(\frac{20000-2000 k}{300 \sqrt{k}}\right)=9.32
$$

The reserves number of air material in typical equipment in 20,000 hours scientifically shoud be 9.32 .

\section{CONCLUSIONS}

Nearly Through making a analysis of the maintenance method of air material in typical equipment, this paper has established consumption models of air material in typical equipment according to stochastic processes and probability theory and mathematical statistics. The models provide a theoretical basis for calculating reserves of air material in typical equipment.

\section{ACKNOWLEDGEMENTS}

This work is supported by supported by the national natural science foundation of China (No. 71401173).

\section{REFERENCES}

[1] LIU Xiao-qun, MA Shi-hua. The Two-hierarchy Stochastic Inventory Model With Multi- varieties [J]. Journal of Huazhong University of Science and Technology, 2005, 33(2): 112-115.

[2] LI Yu-feng, XU Lu-tie, CHEN Yong-kang, ZHAO Ran. Research on Repairable Maintenance Equipment Support of Ground Guidance Kits for a Certain Ammunition [J]. Equipment Environmental Engineering, 2014,11(5):132-136.

[3] WANG Liang, SUN Shao-rong, WU Xiao-ceng. The Twohierarchy Stochastic Inventory Model with Multi-varieties [J].
Journal of Huazhong University of Science and Technology, 2005, 10: 33-38.

[4] WANG Tie-ning, WANG Hong-wei, CAO Yu. Research on Joint Managed Inventory Strategies of Material Supply Chain [J]. China Logistics \& Purchasing, 2009, 13: 56-57.

[5] LI Yu-feng, XU Lu-tie, CHEN Yong-kang, ZHAO Ran. Research on Peace-War Combined Support of Maintenance Equipment of Ground Guidance Kits for a Certain Ammunition [J]. Equipment Environmental Engineering, 2014,11(6):159162. 